\title{
Matrine induces the apoptosis of fibroblast-like synoviocytes derived from rats with collagen-induced arthritis by suppressing the activation of the JAK/STAT signaling pathway
}

\author{
YONGSHENG YANG $^{1,2}$, QIUMEI DONG ${ }^{3}$ and RONGHENG LI ${ }^{1}$ \\ ${ }^{1}$ Department of the Combination of Chinese and Western Medicine, The First Affiliated Hospital of \\ Chongqing Medical University, Chongqing 400016; ${ }^{2}$ Medical Research Center, Southwest Hospital, \\ The Third Military Medical University, Chongqing 400038; ${ }^{3}$ College of Traditional Chinese Medicine, \\ Inner Mongolia Medical University, Hohhot, Inner Mongolia 010000, P.R. China
}

Received April 7, 2016; Accepted November 20, 2016

DOI: $10.3892 /$ ijmm.2016.2843

\begin{abstract}
The induction of apoptosis-resistant rheumatoid synovial tissue cells has been related to constitutively active Janus kinase/signal transducers and activators of transcription (JAK/STAT) signaling in rheumatoid arthritis (RA). The excessive proliferation and inherent resistance to apoptosis of fibroblast-like synoviocytes (FLS) is an important mechanism by which RA originates. However, the effects of matrine on FLS in RA is unclear. The present study aimed to investigate the mechanism of action of matrine in a rat model of collagen-induced arthritis (CIA). The CIA model was established using bovine type II collagen. FLS were isolated from control and CIA rats, cultured in vitro, and confirmed to harbor fibroblast-like characteristics. After treatment of FLS with varying concentrations of matrine, the JAK2 inhibitor AG490, or a combination of both drugs, cell proliferation, apoptosis rate, expression of apoptotic markers and the activation of the JAK/STAT pathway were assessed. Additionally, CIA rats were administered either matrine or methotrexate by oral gavage to examine the effects of therapeutic intervention on arthritis pathogenesis. The arthritis index (AI) was measured and ankle joint structure was analyzed histologically to determine the severity of CIA. Furthermore, expression levels of apoptotic markers and members of the JAK/STAT family were also examined in vivo. Compared with the CIA group, matrine reduced AI and improved ankle pathology. Matrine
\end{abstract}

Correspondence to: Dr Rongheng Li, Department of the Combination of Chinese and Western Medicine, The First Affiliated Hospital of Chongqing Medical University, 1 You Yi Road, Chongqing 400016, P.R. China

E-mail: lrongheng1231@163.com

Key words: matrine, rheumatoid arthritis, fibroblast-like synoviocytes, apoptosis, Janus kinase/signal transducers and activators of transcription signaling pathway, collagen-induced arthritis also inhibited FLS proliferation, induced G0/G1 cell cycle arrest, and increased the rate of apoptosis in vitro. The effects of matrine on apoptosis induction were further confirmed by observations that $\mathrm{Bcl}-2$ levels were decreased, whereas Bax and caspase-3 levels were increased in the matrine-treated synovial tissues and FLS. Finally, matrine treatment also diminished the phosphorylation, and hence activation of JAK2, STAT1 and STAT3. Our results suggest that matrine induces the apoptosis of FLS from rats with CIA by inhibiting activation of the JAK/STAT signaling pathway.

\section{Introduction}

Rheumatoid arthritis (RA), a chronic systemic autoimmune disease primarily affecting the joints, is characterized by synovitis, synovial tissue proliferation, cartilage and bone destruction, and ultimately leads to physical disability (1). RA affects approximately $1 \%$ of the adult population worldwide.

Currently, the exact pathogenesis of RA remains unclear, but accumulating evidence suggests that fibroblast-like synoviocytes (FLS) play an important role in the development of RA because of their resistance to apoptosis and extensive proliferative ability (2). In RA patients, defective apoptosis of synovial cells is closely related to synovial tissue hyperplasia (3). RA-FLS exhibit features of tumor-like damage and erosion, which may lead to further synovial tissue hyperplasia and destruction of joint cartilage (4). Thus, inhibition of RA-FLS proliferation and induction of their apoptosis are under consideration as a therapeutic strategy for the treatment of RA.

The mitochondrial pathway plays a critical role in FLS apoptosis in RA, with the Bax, Bcl-2, and caspase-3 proteins, in particular, being implicated. Bcl-2 and Bax are key regulators of the mitochondrial apoptosis pathway (5). Bax promotes apoptosis, whereas Bcl-2 exerts a wide range of anti-apoptotic effects. Caspase-3 is a key executive mediator of apoptosis and the common downstream effector of many apoptotic signaling pathways.

The Janus kinase/signal transducers and activators of transcription (JAK/STAT) signaling pathway is important for many cellular processes, including growth, differentiation, 
proliferation and immune regulation (6). Aberrant JAK/STAT signaling has been related to the occurrence of many human diseases, including RA and cancer (7). Previous study has indicated that activating JAK/STAT through interferon- $\gamma$ confers apoptotic resistance to cells in synovial tissues of inflammatory RA, leading to a significantly increased density of synovial cells (3). The same study showed that JAK/STAT exerts anti-apoptotic effects via translational regulation (3).

In recent years, JAKs have generated great interest as therapeutic targets given their key roles in signal transduction (8). Activated JAKs trigger a signaling cascade that leads to the phosphorylation of STATs. Phosphorylated STATs form as active dimers, translocate to the nucleus, and bind to specific response elements to activate or inhibit the expression of the target genes. There are seven STAT family members, of which STAT1 and STAT3 represent the major activators (9). STAT1 expression and activity increases in early synovial tissues of human RA, and STAT3 promotes synovial fibroblast survival $(10,11)$. Some studies have suggested that the activation of STAT1 and STAT3 is important to synovial cell proliferation (12).

The traditional approach for controlling RA relies on disease-modifying antirheumatic drugs (DMARDs), including methotrexate (MTX), leflunomide and sulphasalazine. DMARDs have certain clinical benefits, but coincide with a series of toxic events (13). As an alternative treatment for RA, plant extracts are being considered due to lower toxicity profiles and minimal side effects $(14,15)$. Matrine, a traditional Chinese medicine, is an alkaloid isolated from plants in the genus Sophora, comprised of four rings of quinolizidine classes $\left(\mathrm{C}_{15} \mathrm{H}_{24} \mathrm{~N}_{2} \mathrm{O}\right)$. Matrine exhibits different biological properties, including anti-inflammatory (16), antitumor (17), anti-viral (18), anti-fibrosis (19), anti-arrhythmia (20), antiasthmatic (21) and immunosuppressive activity (22). However, the effects of matrine on the proliferation and apoptosis of FLS have yet to be reported.

Collagen-induced arthritis (CIA) is an experimental model of human RA that has been widely used to study the pathogenesis of RA and evaluate the effects of therapeutic interventions $(23,24)$. The purpose of this study was to investigate the effect of matrine on FLS proliferation and apoptosis in a rat model of CIA. We also assessed the mechanisms by which matrine functions to modulate these cellular behaviors.

\section{Materials and methods}

Induction of CIA. Male Sprague-Dawley (SD) rats (7-8 weeks) were purchased from the Experimental Animal Center of the Third Military Medical University (Chongqing, China). Animal care and treatment were completed by the Ethics Committee of the Experimental Animal Administration of the Third Military Medical University. CIA was immune-induced by bovine type II collagen (Chondrex, Inc., Redmond, WA, USA). Type II collagen $(2 \mathrm{mg} / \mathrm{ml})$ dissolved in $0.1 \mathrm{M}$ acetic acid was emulsified with an equal volume of complete Freund's adjuvant (CFA) or incomplete Freund's adjuvant (IFA; MP Biomedicals, Santa Ana, CA, USA). The emulsion (300 $\mu \mathrm{l})$ was injected into the base of the tail on day 0 for the first immunization and on day 7 for the second immunization. An equal volume of saline was administered to rats in the control group at the same time and into the same site.
Culture and drug treatment of FLS. Forty-two days after the initial immunization, randomly chosen rats from the CIA and control groups were sacrificed. Synovial tissues from the knee joints were isolated, minced and cultured in RPMI-1640 medium supplemented with $10 \%$ fetal bovine serum (FBS; Gibco, Grand Island, NY, USA) at $37^{\circ} \mathrm{C}$ in a humidified atmosphere under $5 \% \mathrm{CO}_{2}$. After FLS identification based on cell morphology and immunofluorescent vimentin labeling (25), cells were used from passages 3 to 5 in ensuing experiments. Cell treatments included the JAK2 inhibitor, AG490 $(25 \mu \mathrm{mol} / \mathrm{l}$; Abmole Bioscience, Houston, TX, USA) or matrine $(0.75 \mathrm{mg} / \mathrm{ml}$; Aladdin Reagents Co., Ltd., Shanghai, China).

Cell proliferation assay. Cell proliferation was analyzed using the Cell Counting Kit-8 (CCK-8; Dojindo Laboratories, Kumamoto, Japan). Cells were seeded in three 96-well plates at a density of $3 \times 10^{4}$ cells/well and treated with increasing concentrations $(0,0.25,0.5,0.75,1.0,1.5$ and $2.0 \mathrm{mg} / \mathrm{ml})$ of matrine for 24,48 and $72 \mathrm{~h}$. Then, $10 \mu \mathrm{l}$ of CCK- 8 solution was added to each well, and the cells were incubated for another $2 \mathrm{~h}$ at $37^{\circ} \mathrm{C}$. The optical density (OD) was measured using a microplate reader (Beijing Purkinje General Instrument Co., Ltd., Beijing, China) at a wavelength of $450 \mathrm{~nm}$. The cell growth inhibition rate (GIR) was measured and the half maximal inhibitory concentration $\left(\mathrm{IC}_{50}\right)$ was calculated using GraphPad Prism 5 (GraphPad Software, San Diego, CA, USA).

Cell cycle analysis. FLS were collected in the logarithmic growth phase and seeded in culture flasks at a density of $2 \times 10^{5}$ cells $/ \mathrm{ml}$. After synchronization in serum-free medium for $24 \mathrm{~h}, \mathrm{FLS}$ were treated with matrine, AG490, matrine+AG490, or left untreated for $24 \mathrm{~h}$. After drug treatment, the cells were collected, suspended in cold phosphate-buffered saline (PBS), centrifuged, and then fixed with $75 \%$ cold ethanol and stored at $4^{\circ} \mathrm{C}$ for $24 \mathrm{~h}$. The cells were stained with propidium iodide (PI) solution (Cell Cycle and Apoptosis Analysis kit; Beyotime Institute of Biotechnology, Nanjing, China) for $30 \mathrm{~min}$ at $37^{\circ} \mathrm{C}$ in the dark. DNA content was analyzed by flow cytometry (Beckman Coulter, Brea, CA, USA).

Apoptosis analysis. FLS apoptosis was assessed with the Annexin V-FITC/PI double labeling method combined with flow cytometry. Briefly, the cells were seeded at a density of $2 \times 10^{5}$ cells $/ \mathrm{ml}$ in five culture flasks for $24 \mathrm{~h}$ and synchronized. After exposure to drugs for $24 \mathrm{~h}$, the cells were collected, re-suspended in PBS, counted, centrifuged and resuspended in $195 \mu \mathrm{l}$ binding buffer according to the manufacturer's instructions. The cell suspension was incubated in $5 \mu \mathrm{l}$ of Annexin V-FITC and $10 \mu 1$ PI (Annexin V-FITC Apoptosis Detection kit; Beyotime Institute of Biotechnology) for $20 \mathrm{~min}$ at room temperature in the dark. Apoptotic cells were analyzed by flow cytometry.

Western blot analysis. The cells were collected in radioimmunoprecipitation assay (RIPA) lysis buffer (Chongqing Golden Wheat Biotechnology, Chongqing, China). The protein concentration was determined using the bicinchoninic acid (BCA) protein assay (Beyotime Institute of Biotechnology). Proteins were separated using sodium dodecyl sulfate-polyacrylamide gel electrophoresis (SDS-PAGE) and 
Table I. Primers used for qRT-PCR analysis.

\begin{tabular}{lll}
\hline Genes & \multicolumn{1}{c}{ Primer sequence (5'-3') } & Accession no. \\
\hline Bax & $\begin{array}{l}\text { F: GGCGATGAACTGGACAACAA } \\
\text { R: GCAAAGTAGAAAAGGGCAACC }\end{array}$ & NM 017059.2 \\
Bcl-2 & F: ACGAGTGGGATACTGGAGATGA & NM 016993.1 \\
& R: CTCAGGCTGGAAGGAGAAGAT & \\
Caspase-3 & F: ATCCACGAGCAGAGTCAAAGG & NM 012922.2 \\
& R: CAAGCCAACCAAGTTCACACA & \\
$\beta$-actin & F: CGTAAAGACCTCTATGCCAACA & NM 031144.3 \\
& R: AGCCACCAATCCACACAGAG & \\
\hline
\end{tabular}

F, forward; R, reverse.

transferred to polyvinylidene difluoride (PVDF) membranes. Then, the membranes were blocked with 5\% skim milk for $2 \mathrm{~h}$ at room temperature. The membranes were incubated overnight at $4^{\circ} \mathrm{C}$ in antibodies against $\beta$-actin (1:1,000; Santa Cruz Biotechnology, Inc., Santa Cruz, CA, USA), p-JAK2 (1:1000; Nanjing Jiancheng Bioengineering Institute, Nanjing, China), p-STAT1 and p-STAT3 (1:1,000; both from BBI Life Sciences Corporation, Shanghai, China). The next day, the membranes were washed three times in Tris-buffered saline containing $0.05 \%$ Tween-20 (TBST) and incubated in horseradish peroxidase-conjugated anti-rabbit secondary antibody $(1: 1,000$; Beyotime Institute of Biotechnology) for $2 \mathrm{~h}$ at room temperature. The protein bands were visualized with ECL reagents (Beyotime Institute of Biotechnology).

For the rat experiments, synovial tissues were isolated and ground into a powder with liquid nitrogen. Proteins were collected in RIPA buffer, and the expression levels of phosphorylated JAK2, STAT1 and STAT3 were detected by western blot analysis as described above.

Quantitative real-time PCR ( $q R T-P C R)$. Total RNA was extracted from cultured FLS with TRIzol reagent according to the manufacturer's instructions (Sangon Biotech Co., Ltd., Shanghai, China). The RNA was reverse-transcribed with AMV First Strand cDNA Synthesis kit (Sangon Biotech Co., Ltd.) in the presence of random primers and RNase-free $\mathrm{ddH}_{2} \mathrm{O}$. The primers were designed by Sangon Biotech Co., Ltd. and are listed in Table I. $\beta$-actin was used as an endogenous control. The conditions for the amplifications were as follows: $3 \mathrm{~min}$ hold at $95^{\circ} \mathrm{C}$, followed by $7 \mathrm{sec}$ denaturation at $95^{\circ} \mathrm{C}, 10 \mathrm{sec}$ annealing at $57^{\circ} \mathrm{C}$, and $15 \mathrm{sec}$ extension at $72^{\circ} \mathrm{C}$ for 40 cycles. The PCR products were analyzed using StepOne Plus ${ }^{\mathrm{TM}}$ Real-Time PCR system (Applied Biosystems, Grand Island, NY, USA). The qRT-PCR data were analyzed using the $2^{-\triangle \Delta C T}$ method.

CIA drug treatments. Rats with CIA were divided randomly into a control group, CIA group, matrine group [100 mg/kg/day by oral gavage (19)], and MTX group [Shanghai Sine Pharmaceuticals Co., Ltd., Shanghai, China; $2 \mathrm{mg} /$ $\mathrm{kg} /$ week by oral gavage (26)]. Control and CIA rats received saline at a dose of $10 \mathrm{ml} / \mathrm{kg} /$ day by oral gavage. Drugs were administered from days 11 to 52 after the first immunization.
Evaluation of arthritis index (AI). AI was assessed by the presence of edema and/or erythema in the paws that started before gavage, occurred once a week, and lasted for six weeks. Assessment standards have been previously described (27) as follows: 0 , no redness or swelling; 1 , light redness or swelling in a few toes; 2 , redness and swelling in most toe joints and toes; 3 , serious redness and swelling in feet and below ankle joint; and 4, redness and swelling in feet and ankle joint. The AI was calculated by cumulation of the scores from all four paws, with a maximum value of 16 .

The pathological alteration of ankle joints. Rats received matrine or MTX over the course of six weeks before they were sacrificed by cervical dislocation. The ankles were removed and fixed in 4\% paraformaldehyde (Guangfu Fine Chemical Research Institute, Tianjin, China) and decalcified for $16 \mathrm{~h}$ with $8 \%$ nitrate decalcification solution (Chengdu Kelong Chemical Co., Ltd., Chengdu, China). Then, the joints were washed, dehydrated, brightened, embedded in paraffin, sectioned and stained with hematoxylin and eosin (H\&E). Pathological alterations were detected with a light microscope (BX51M; Olympus, Tokyo, Japan).

Immunohistochemical analysis. The rats were sacrificed and then their synovial tissues were isolated from their knee joints and embedded in paraffin. Sections (5- $\mu \mathrm{m}$ thick) were cut on a microtome and then dewaxed in water. Endogenous peroxidases were inactivated and antigen retrieval was conducted using a boiling method. Then, the sections were blocked in goat serum for $15 \mathrm{~min}$ at room temperature and incubated overnight at $4^{\circ} \mathrm{C}$ with antibodies against Bax (1:100) and Bcl-2 (1:100) (both from BBI Life Science Corporation), and caspase-3 (1:100; Nanjing Jiancheng Bioengineering Institute). A biotin-labeled secondary antibody and SABC solution were added dropwise, successively and incubated for $20 \mathrm{~min}$ at room temperature. The sections were developed with DAB, re-stained with hematoxylin, dehydrated, brightened and mounted with neutral gum. Images were captured on a light microscope (Olympus) and OD values were calculated with IPP software.

Statistical analysis. Quantitative data are presented as mean \pm standard deviation (SD). All data were analyzed using SPSS 19.0 software (SPSS, Inc., Chicago, IL, USA). Statistical significance was assessed by one-way analysis of variance (ANOVA) or the Games-Howell test according to the homogeneity of variance. $\mathrm{P}$-values $<0.05$ were considered to indicate a statistically significant difference.

\section{Results}

Identification of FLS. By passage three, the cellular morphology of cultured rat FLS appeared spindle-shaped, as is characteristic of fibroblasts (Fig. 1A). In addition, $100 \%$ of the cultured cells expressed vimentin as detected by immunofluorescence, thus confirming FLS identity (Fig. 1B).

Matrine inhibits the proliferation of rat-derived FLS in vitro. CCK-8 assay assessment of cell viability of the FLS isolated from the CIA rats treated with varying concentrations $(0$, $0.25,0.5,0.75,1.0,1.5$ and $2.0 \mathrm{mg} / \mathrm{ml}$ ) of matrine showed that 


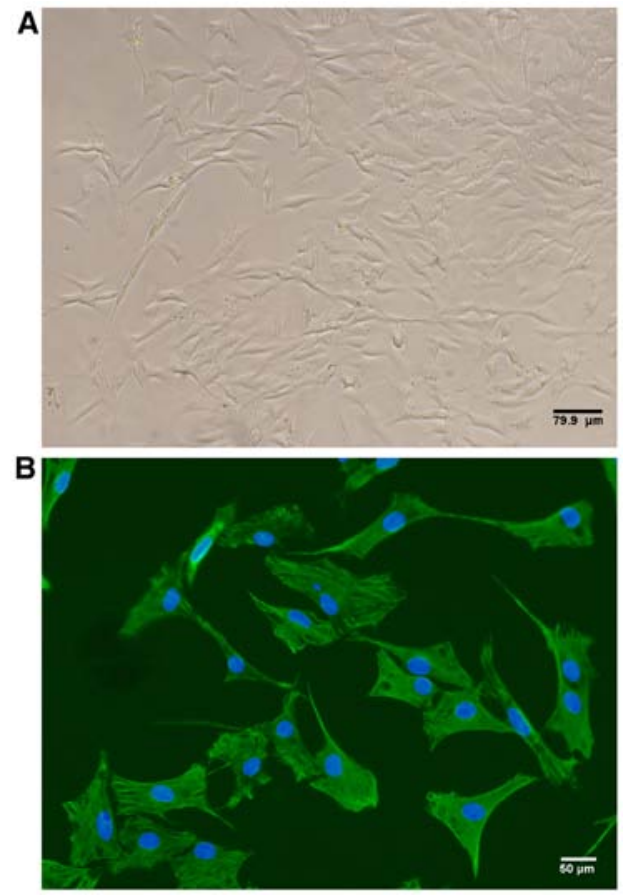

Figure 1. Fibroblast-like synoviocytes (FLS) were isolated from the knee joints of rats with collagen-induced arthritis (CIA) 42 days after the initial immunization. (A) Representative phase contrast image exhibiting typical morphology of FLS. Scale bar, $79.9 \mu \mathrm{m}$. (B) Representative immunofluorescence image of FLS stained with vimentin (green). Nuclei were stained with DAPI (blue). Scale bar, $50 \mu \mathrm{m}$.

matrine inhibited FLS growth in a concentration-dependent manner at 24,48 and $72 \mathrm{~h}(\mathrm{P}<0.01$; Fig. 2$)$. The $\mathrm{IC}_{50}$ of matrine inhibitory activity was estimated to be $0.75 \mathrm{mg} / \mathrm{ml}$ at $24 \mathrm{~h}$, and these parameters were used in subsequent experiments.

Matrine induces cell cycle arrest of rat-derived FLS in vitro. Flow cytometry of FLS revealed that the percentage of cells in $\mathrm{S}$ phase was increased $(\mathrm{P}<0.01)$, whereas the percentage of cells in the G0/G1 phase was decreased $(\mathrm{P}<0.05)$ in the CIA group compared with the control group (Fig. 3). Compared with the CIA group, the percentages of FLS in the S phase were significantly decreased in cells treated with matrine, the specific JAK2 inhibitor AG490 (inhibits phosphorylation of STAT1/3 in RA-FLS), or both for $24 \mathrm{~h}(\mathrm{P}<0.01$; Fig. 3). Additionally, the percentages of cells in the G0/G1 phase were also increased in the drug-treated groups compared with CIA alone $(\mathrm{P}<0.01)$. The percentage of cells in the $\mathrm{S}$ phase in the AG490-treated group was less than that in the matrine group $(\mathrm{P}<0.01)$, but greater than that in the matrine+AG490 group $(\mathrm{P}<0.05)$ (Fig. 3). These findings suggest that matrine inhibits cell proliferation by inducing cell cycle arrest at the G0/G1 phase.

Matrine induces apoptosis of rat-derived FLS in vitro. Annexin V-FITC/PI double staining and flow cytometry indicated that the apoptosis rate of FLS isolated from control rats was $17.24 \pm 0.24 \%$ compared with $13.64 \pm 0.49 \%$ in the CIA group ( $\mathrm{P}<0.01$; Fig. 4). All drug treatments resulted in significantly higher apoptosis rates than that observed for the CIA group $(\mathrm{P}<0.01$; matrine, 20.10 $\pm 0.77 \%$; AG490, 20.42 $\pm 0.91 \%$; matrine+AG490, 25.11 $\pm 1.00 \%)$. The apoptosis rate did not

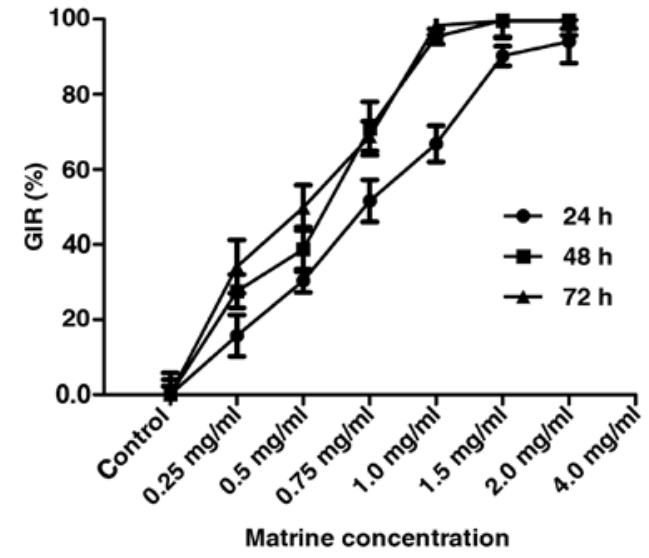

Figure 2. The effect of matrine on fibroblast-like synoviocyte (FLS) growth inhibition was measured by CCK-8 assay. FLS were isolated from rats with collagen-induced arthritis (CIA), cultured in vitro, and treated with various concentrations of matrine for 24,48 and $72 \mathrm{~h}$. Matrine significantly reduced the growth inhibition rate (GIR) of FLS in a concentration-dependent manner. Treatment of cells with $0.75 \mathrm{mg} / \mathrm{ml}$ matrine for $24 \mathrm{~h}$ led to a nearly $50 \%$ decrease in cell growth.

differ between the matrine and AG490 groups. These results indicate that matrine treatment increased FLS apoptosis.

Matrine suppresses JAK/STAT signaling pathway activation in rat-derived FLS in vitro. Protein expression levels of p-JAK2, p-STAT1 and p-STAT3 in FLS isolated after $24 \mathrm{~h}$ of drug treatment were only faintly detectable in the control group, and markedly higher in the CIA group ( $\mathrm{P}<0.01$ vs. control; Fig. 5). All phosphorylated protein levels were decreased relative to CIA alone when FLS from CIA rats were treated with matrine (p-JAK2, P<0.01; p-STAT1 and p-STAT3, P<0.05), AG490 (all $\mathrm{P}<0.01$ ), or matrine+AG490 (all $\mathrm{P}<0.01$ ). In addition, p-JAK2 and p-STAT3 were inhibited to a greater extent by treatment with AG490 than by treatment with matrine $(\mathrm{P}<0.05)$. However, $\mathrm{p}-\mathrm{STAT} 1$ levels did not differ between the matrine and AG490 groups ( $\mathrm{P}>0.05)$.

Matrine induces upregulation of pro-apoptotic markers in rat-derived FLS in vitro. As shown in Fig. 6, qRT-PCR analysis revealed that the expression levels of Bax and caspase-3 in the CIA group were decreased, whereas Bcl-2 expression was increased, compared to levels observed for the control group FLS $(\mathrm{P}<0.01)$. Treatment with matrine, AG490, or matrine+AG490 markedly increased the mRNA expression of Bax and caspase-3, and decreased expression of $\mathrm{Bcl}-2$ ( $\mathrm{P}<0.01$ vs. CIA alone). Matrine treatment resulted in higher levels of Bcl-2 (Fig. 6B) and lower levels of caspase-3 (Fig. 6C) than did the AG490 treatment $(\mathrm{P}<0.05)$. Bax levels did not differ between the matrine and AG490 groups ( $\mathrm{P}>0.05$; Fig. 6A). Finally, the Bax:Bcl-2 ratio was increased in all drug-treated groups compared with CIA alone $(\mathrm{P}<0.01$; Fig. 6D).

Therapeutic effects of matrine in rats with CIA. Evaluation of AI and pathological alterations of the ankle to determine arthritis severity in the CIA model indicated that onset of secondary arthritis appeared around day 10 after primary 

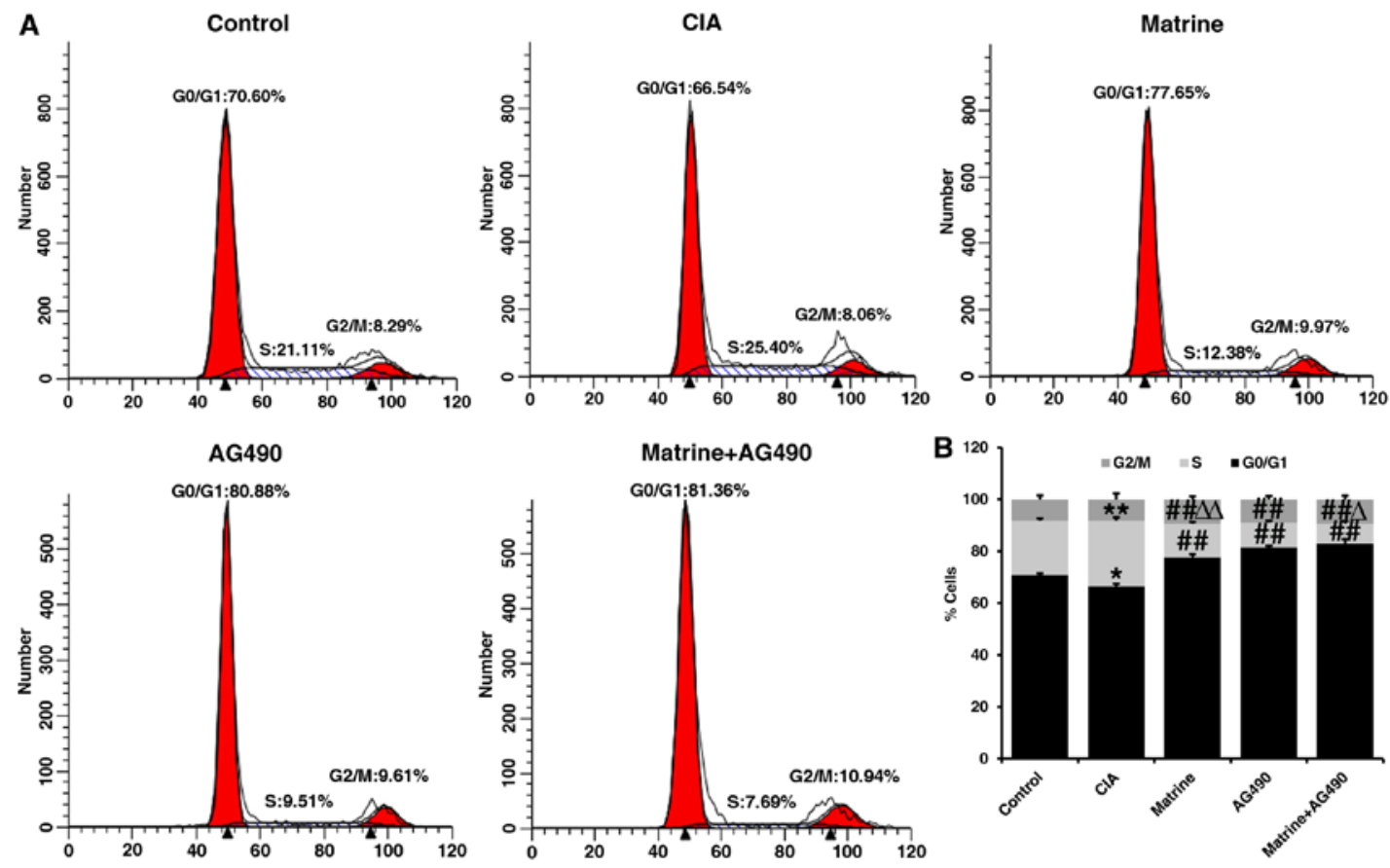

Figure 3. The effect of matrine on cell cycle progression was determined by propidium iodide (PI) staining and flow cytometry. Fibroblast-like synoviocytes (FLS) were isolated from control or collagen-induced arthritis (CIA) rats, cultured in vitro, and treated with $0.75 \mathrm{mg} / \mathrm{ml} \mathrm{matrine,} 25 \mu \mathrm{mol} / 1 \mathrm{AG} 490$, or a combination of matrine and AG490 for $24 \mathrm{~h}$. (A) Cell cycle distribution on FLS after $24 \mathrm{~h}$ of drug treatment. (B) Histogram depicts the percentage of cells in $\mathrm{G} 0 / \mathrm{G} 1, \mathrm{~S}$ and $\mathrm{G} 2 / \mathrm{M}$ phases of the cell cycle. Data are expressed as mean $\pm \mathrm{SD}$ of three independent experiments. ${ }^{*} \mathrm{P}<0.05$, ${ }^{* *} \mathrm{P}<0.01 \mathrm{vs}$. the control group; ${ }^{\# \#} \mathrm{P}<0.01$ vs. the CIA group; ${ }^{\Delta} \mathrm{P}<0.05,{ }^{\Delta \Lambda} \mathrm{P}<0.01$ vs. the AG490 group.
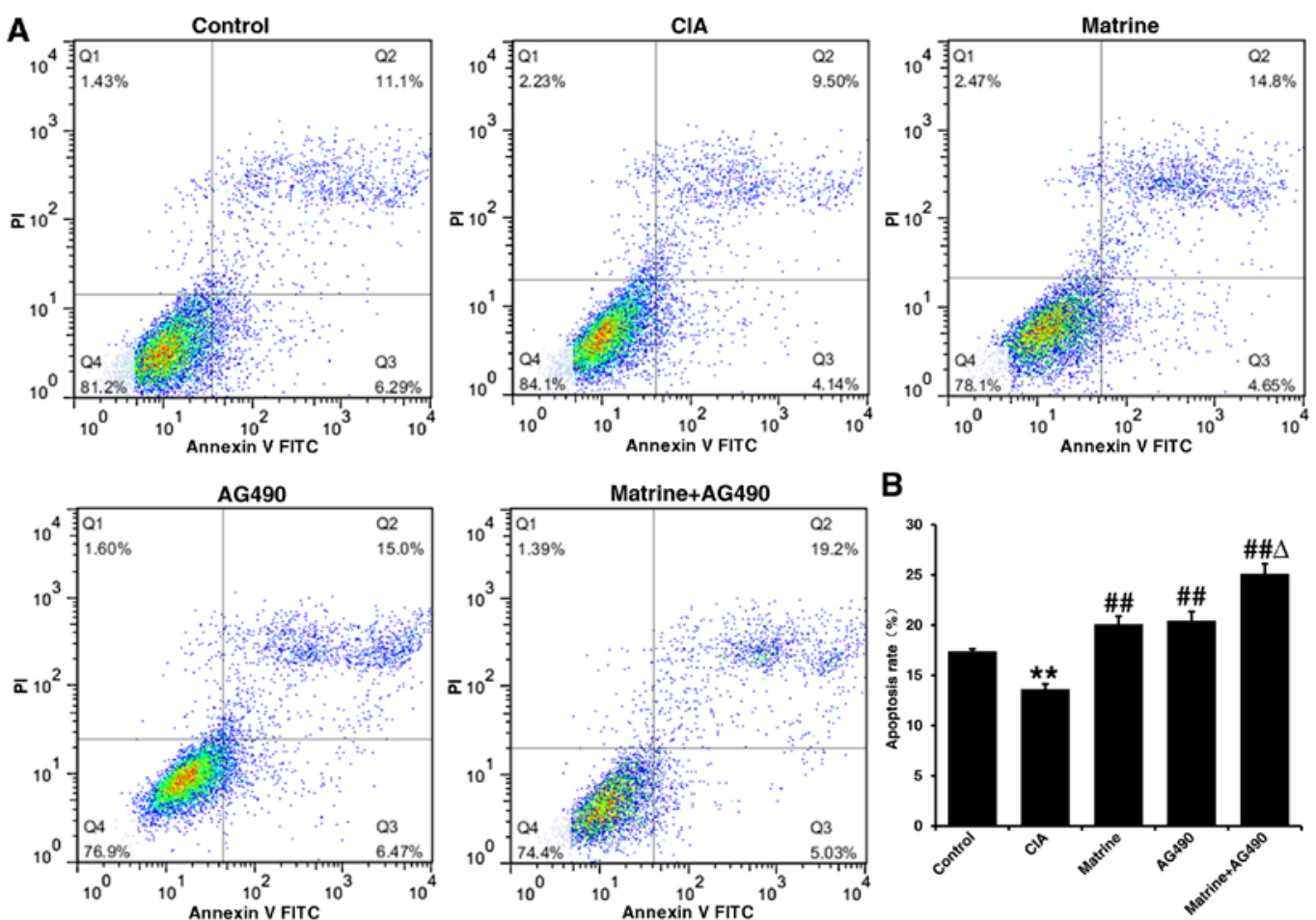

Figure 4. The effect of matrine on apoptosis was determined by double staining with Annexin V-FITC/propidium iodide (PI) and flow cytometry. Fibroblast-like synoviocytes (FLS) were isolated from control or collagen-induced arthritis (CIA) rats, cultured in vitro, and treated with $0.75 \mathrm{mg} / \mathrm{ml} \mathrm{matrine}, 25 \mu \mathrm{mol} / \mathrm{l}$ AG490, or a combination of matrine and AG490 for 24 h. (A) Representative FACS scatter plots for each drug treatment. Q1 indicates necrotic cells; Q2 indicates late apoptotic cells; Q3 indicates early apoptotic cells; and Q4 indicates living cells. (B) Cell apoptosis rates were analyzed via flow cytometry. Data are presented as mean $\pm \mathrm{SD}$ of three independent experiments. ${ }^{* *} \mathrm{P}<0.01$ vs. the control group; ${ }^{\# \#} \mathrm{P}<0.01$ vs. the CIA group; ${ }^{\Delta} \mathrm{P}<0.05$ vs. the AG490 group.

immunization and peaked on day 31. Matrine treatment decreased AI compared with no treatment $(\mathrm{P}<0.05$; Fig. 7A).
There were no statistically significant differences between the matrine and MTX groups $(\mathrm{P}>0.05)$. 

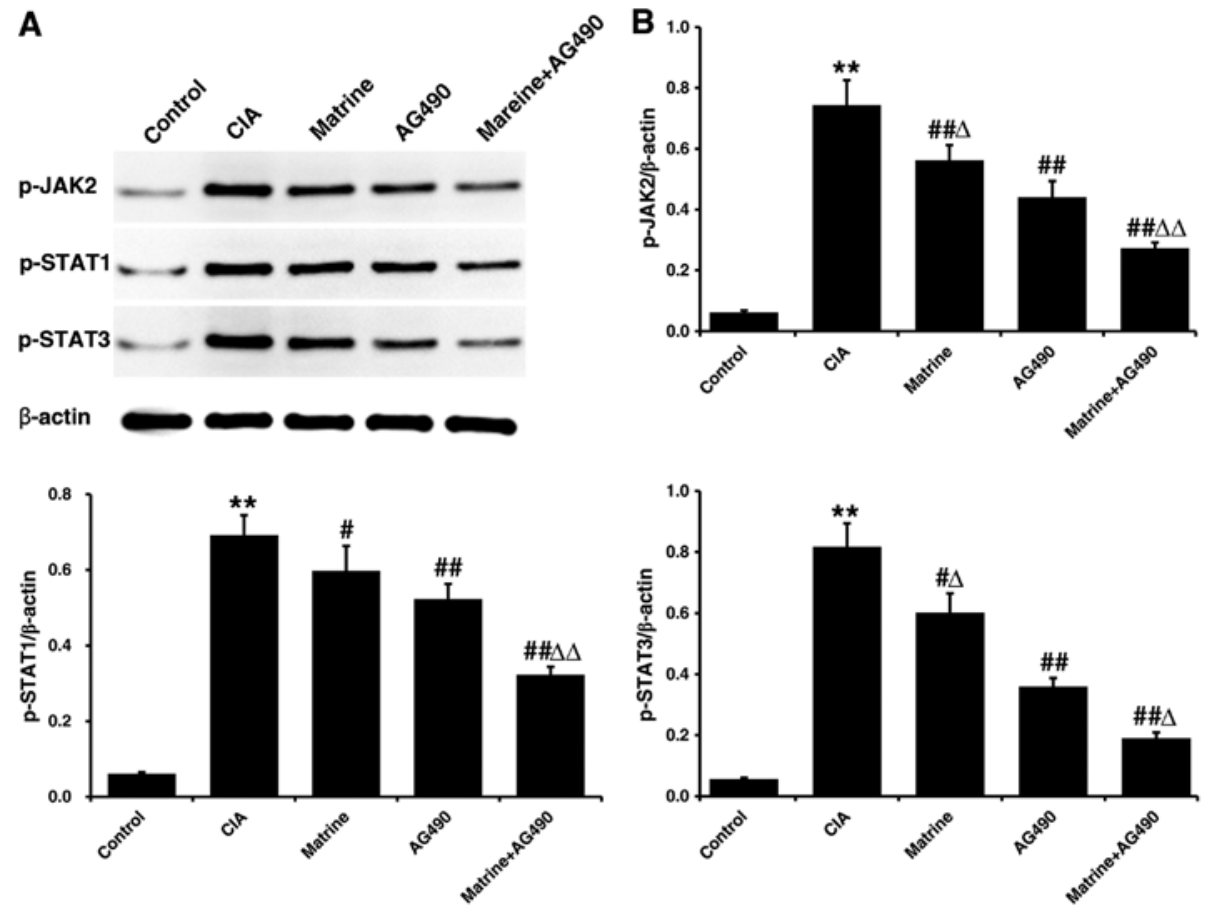

Figure 5. The effects of matrine on the Janus kinase/signal transducers and activators of transcription (JAK/STAT) pathway were assessed by western blot analysis. Fibroblast-like synoviocytes (FLS) were isolated from control or collagen-induced arthritis (CIA) rats, cultured in vitro, and treated with $0.75 \mathrm{mg} / \mathrm{ml}$ matrine, $25 \mu \mathrm{mol} / 1$ AG490, or a combination of matrine and AG490 for $24 \mathrm{~h}$. (A) Western blot analysis of p-JAK2, p-STAT1 and p-STAT3 levels. $\beta$-actin was used as a loading control. (B) Quantitative densitometry of p-JAK2, p-STAT1 and p-STAT3 levels relative to $\beta$-actin. Data are presented as mean \pm SD of three independent experiments. ${ }^{* *} \mathrm{P}<0.01$ vs. the control group; ${ }^{\#} \mathrm{P}<0.05,{ }^{\# \#} \mathrm{P}<0.01$ vs. the CIA group; ${ }^{\Delta} \mathrm{P}<0.05,{ }^{\Delta \Delta} \mathrm{P}<0.05$ vs. the AG490 group.
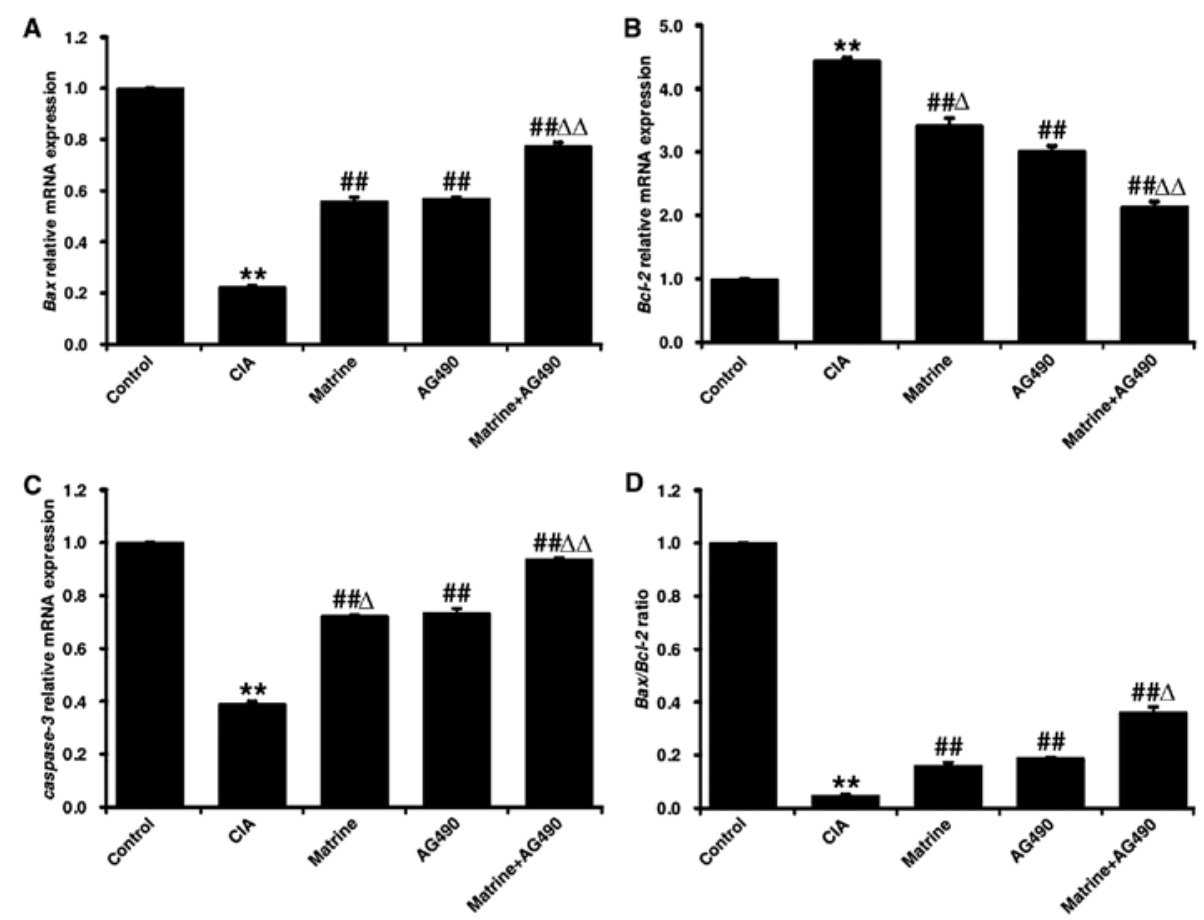

Figure 6. The effects of matrine on apoptotic markers were assessed by qRT-PCR. Fibroblast-like synoviocytes (FLS) were isolated from control or collagen-induced arthritis (CIA) rats, cultured in vitro, and treated with $0.75 \mathrm{mg} / \mathrm{ml}$ matrine, $25 \mu \mathrm{mol} / \mathrm{l} \mathrm{AG} 490$, or a combination of matrine and AG490 for $24 \mathrm{~h}$. qRT-PCR analysis of relative expression of (A) Bax, (B) Bcl-2 and (C) caspase-3. (D) Bax to Bcl-2 ratio (based on relative intensity). In all experiments, $\beta$-actin was used as an internal control. Data are presented as the mean $\pm \mathrm{SD}$ of three independent experiments. ${ }^{* *} \mathrm{P}<0.01$ vs. the control group; ${ }^{\# / t} \mathrm{P}<0.01 \mathrm{vs}$. the CIA group; ${ }^{{ }^{\mathrm{P}}} \mathrm{P}<0.05,{ }^{\Delta \Delta} \mathrm{P}<0.01$ vs. the $\mathrm{AG} 490$ group.

Microscopic assessment of ankle structure revealed that control rats displayed smooth articular surfaces with no evidence of inflammatory cell infiltration or cartilage destruction (Fig. 7B). In contrast, the ankles of rats in the CIA group 


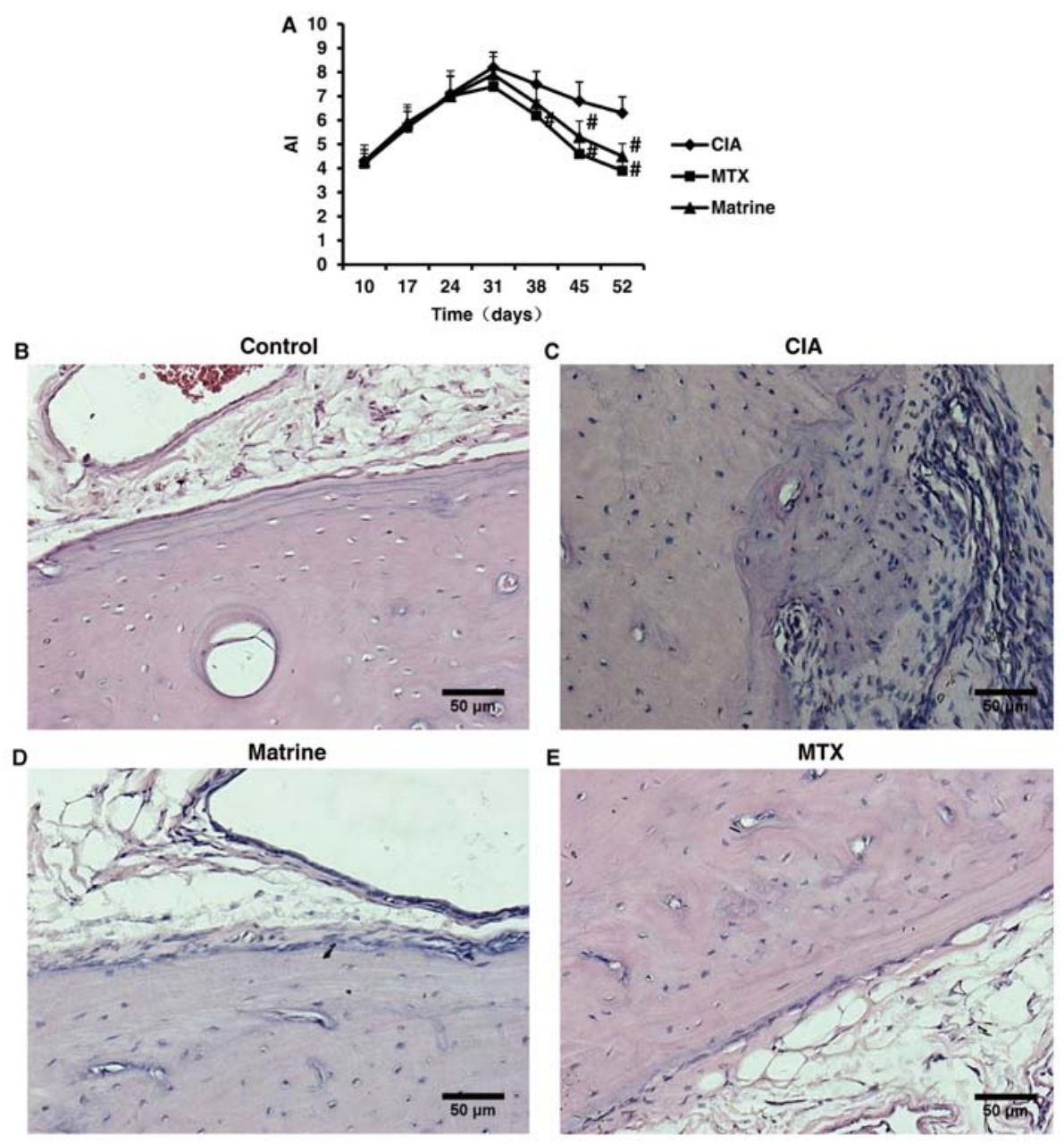

Figure 7. The therapeutic effects of matrine on collagen-induced arthritis (CIA) were assessed by arthritis index (AI) and ankle pathological analysis in control rats and CIA rats treated with $100 \mathrm{mg} / \mathrm{kg}$ matrine or $2 \mathrm{mg} / \mathrm{kg}$ methotrexate (MTX). (A) AI over 52 days of drug treatment (n=10 rats). Data are presented as mean \pm SD. ${ }^{~} \mathrm{P}<0.05$ vs. CIA group. (B-E) Representative H\&E images of synovial tissues isolated from ankle joints. (B) Control animals exhibited smooth articular surfaces and no signs of inflammatory cell infiltration or cartilage destruction. (C) Rats with CIA displayed synovial hyperplasia, extensive inflammatory cell infiltration and cartilage destruction. (D) Rats in the CIA group treated with matrine exhibited slight synovial tissue hyperplasia, fewer immune infiltrates and no cartilage erosion or bone destruction. (E) Rats in the CIA group treated with MTX also displayed fewer immune infiltrates, and no signs of synovial hyperplasia or cartilage and bone destruction. All images were captured at x200 magnification.

exhibited significant synovial tissue hyperplasia, inflammatory cell infiltration and cartilage erosion (Fig. 7C). Treatment with matrine or MTX improved CIA ankle pathologies (Fig. 7B-D).

Effects of matrine on apoptotic marker expression in synovial tissues from CIA rats. Immunohistochemical analysis demonstrated Bax, Bcl-2 and caspase- 3 expression in the nucleus and cytoplasm in synovial tissue cells (positivity indicated with tan granules; Fig. 8A-C). Quantification of immunohistochemical OD values revealed that the expression of Bax and caspase-3 were decreased, whereas $\mathrm{Bcl}-2$ expression was increased in the CIA group compared with the control group $(\mathrm{P}<0.01)$. Treatment with matrine or MTX rescued Bax and caspase-3 levels and attenuated $\mathrm{Bcl}-2$ expression, but matrine had greater effects on reduction of Bax and caspase- $3(\mathrm{P}<0.01)$ and augmentation of Bcl-2 $(\mathrm{P}<0.05)$ relative to MTX (Fig. 8D-F). Finally, the Bax:Bcl-2 ratio was increased in the matrine group compared with CIA alone ( $\mathrm{P}<0.01$; Fig. 8G).

Effects of matrine on JAK/STAT signaling in synovial tissues from rats with CIA. Phosphorylated JAK2, STAT1 and STAT3 levels were examined in synoviocytes from rats with CIA by western blot analysis. Protein expression levels of p-JAK2, p-STAT1 and p-STAT3 were detected at low levels in the control rats, whereas CIA resulted in increased phosphorylation $(\mathrm{P}<0.01$; Fig. 9). Matrine treatment reduced phosphorylated levels of all three proteins compared with CIA alone $(\mathrm{P}<0.01)$. MTX treatment was more potent than matrine at reducing phosphorylation $(\mathrm{P}<0.01)$.

\section{Discussion}

In the present study, we investigated the effects of matrine treatment on the proliferation, apoptosis and activation of JAK/STAT signaling in FLS isolated from rats with CIA. We found that matrine exerted therapeutic effects on CIA, inhibited FLS proliferation, and induced apoptosis at least partly through downregulation of JAK/STAT signaling.

$\mathrm{RA}$ is a heterogeneous disease characterized by prominent synoviocyte hyperplasia and a potential imbalance between FLS overgrowth and apoptosis (28). RA pathogenesis is affected by multiple etiologies, including genetic, epigenetic and 

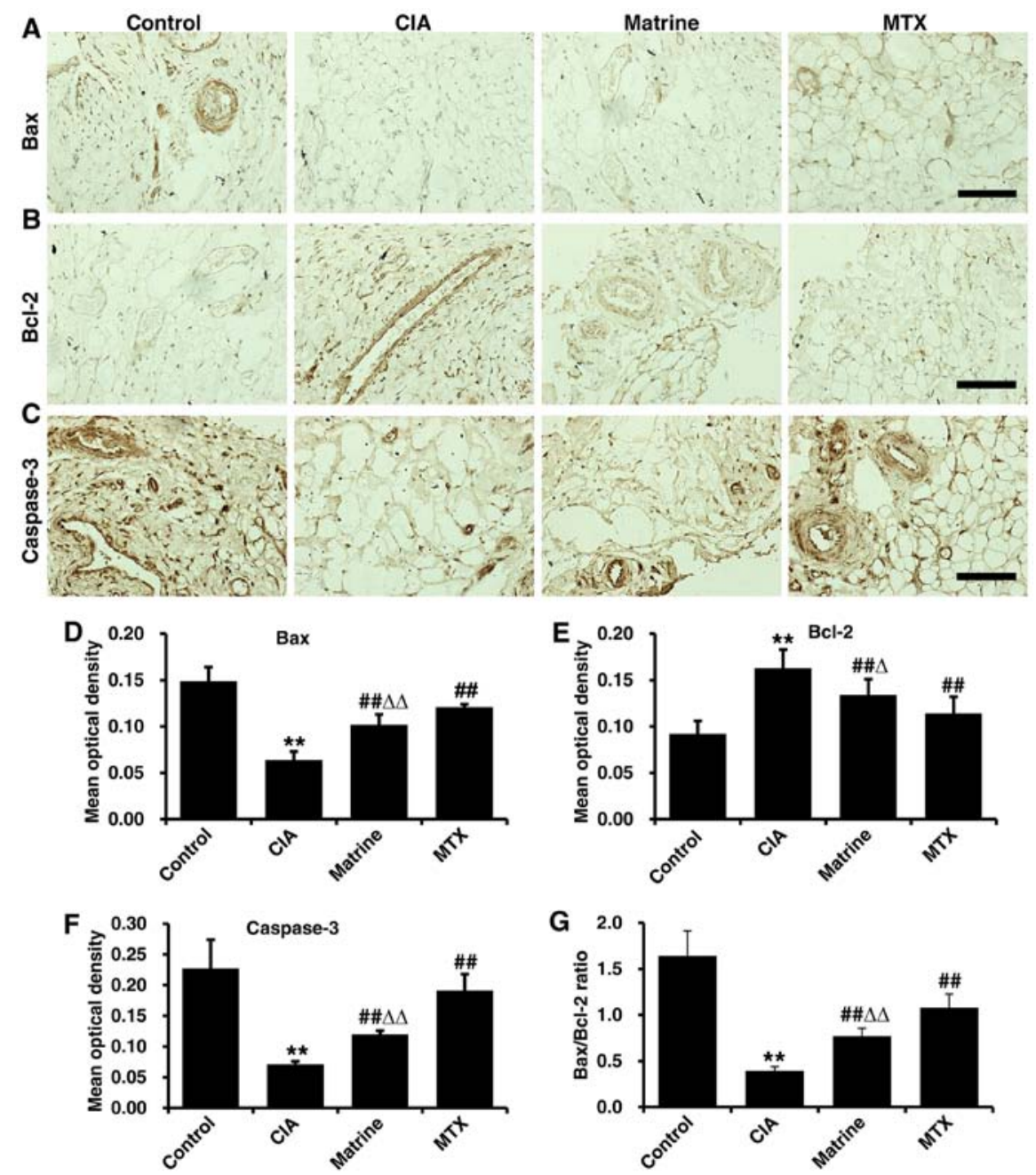

Figure 8. The effects of matrine on the expression of apoptotic markers in synovial tissues were detected by immunohistochemistry. Rats with collagen-induced arthritis (CIA) were treated with $100 \mathrm{mg} / \mathrm{kg}$ matrine or $2 \mathrm{mg} / \mathrm{kg}$ methotrexate (MTX). Representative immunohistochemistry images of (A) Bax, (B) Bcl-2 and (C) caspase-3. Scale bars, $50 \mu \mathrm{m}$. (D-F) Immunohistochemistry signals were quantified by measuring the mean optical density (OD) of each image. (G) Bax to $\mathrm{Bcl}-2$ ratio based on mean OD values. Data are presented as mean OD values (mean $\pm \mathrm{SD})$ for each group $(\mathrm{n}=10)$. ${ }^{* *} \mathrm{P}<0.01$ vs. the control group; ${ }^{\# \#} \mathrm{P}<0.01$ vs. the CIA group; ${ }^{\Delta \Delta} \mathrm{P}<0.05$ vs. the MTX group.

A
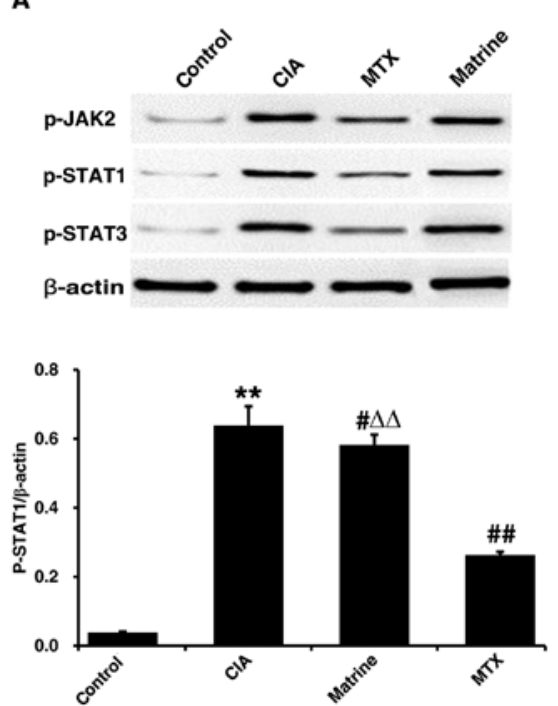

B
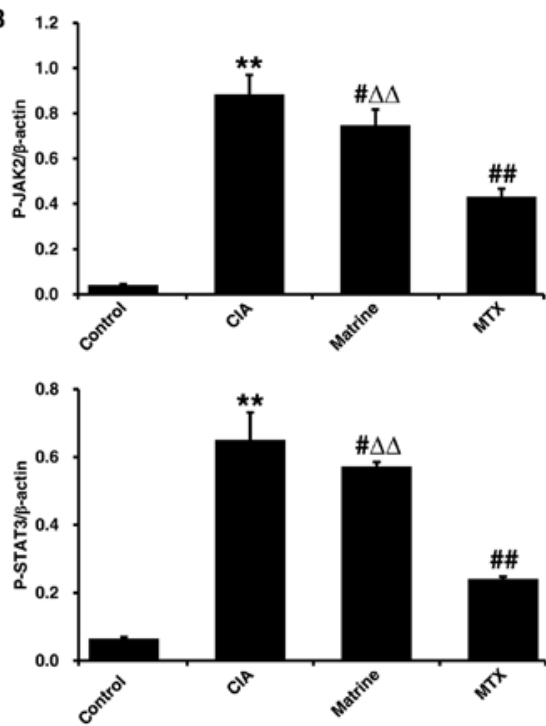

Figure 9. The effects of matrine on the Janus kinase/signal transducers and activators of transcription (JAK/STAT) pathway were determined by western blot analysis in rats sacrificed on day 53 after the initial immunization. Rats with collagen-induced arthritis (CIA) were treated with $100 \mathrm{mg} / \mathrm{kg} \mathrm{matrine} \mathrm{or} 2 \mathrm{mg} / \mathrm{kg}$ methotrexate (MTX). (A) Western blot analysis of p-JAK2, p-STAT1 and p-STAT3 levels. $\beta$-actin was used as a loading control. (B) Quantitative densitometry of p-JAK2, p-STAT1 and p-STAT3 levels relative to $\beta$-actin. Data are presented as the means \pm SD of three independent experiments. ${ }^{* *} \mathrm{P}<0.01$ vs. the control group; ${ }^{*} \mathrm{P}<0.05,{ }^{\# \#} \mathrm{P}<0.01$ vs. the CIA group; ${ }^{\Delta \Delta} \mathrm{P}<0.05$ vs. the MTX group. 
environmental factors, as well as by immune disturbance and cytokine factors. DMARDs, non-steroidal anti-inflammatory drugs (NSAIDs), steroid hormones, and biologics are typically used to treat RA, but are associated with severe adverse effects, including gastrointestinal lesions, cardiovascular complications, and reproductive toxicity (29). Therefore, the exploitation of plant-derived drugs as anti-RA agents with potent effects and lower toxicity profiles has drawn much attention (30). Matrine is the primary active component of the traditional Chinese medicinal herb Sophora flavescens Ait. and is associated with cell growth inhibition and apoptosis induction in cancer cells $(17,31)$. FLS exhibit proliferative, aggressive, and tumor-like behaviors in patients with RA (28), and may therefore be optimal targets for matrine activity. While some of the effects of matrine are related to the etiological factors underlying RA, the mechanism of action of this compound remains uncharacterized.

The pathological changes to the blood and articular tissue in the CIA model are similar to those in human RA, highlighting the value of this system for studying human arthritis (32). In this study, we assessed the effects of matrine on arthritis severity in rats using AI and ankle pathological analysis and found that matrine reduced AI and improved ankle structure. Our in vitro experiments clearly indicated that matrine markedly inhibited cell proliferation in a concentration-dependent manner, blocked G0/G1 cell cycle progression, and induced apoptosis in FLS derived from rats with CIA. These findings demonstrated for the first time that matrine can trigger FLS apoptosis, which may reflect a mechanism of action related to its anti-RA effects.

Increasing evidence suggests that a reduced level of apoptosis in vivo is closely associated with FLS proliferation in RA (33). Apoptosis is a distinct form of cell death initiated by various physiological and pathological stimuli (34). The Bcl-2 family consists of key regulatory proteins involved in mitochondrial apoptotic pathways (2). A decrease in Bcl-2 levels or an increase in Bax levels can trigger signals to initiate the apoptotic cascade (33). Various studies have examined the relative expression status of Bcl-2 family members in RA (35). Pro-apoptotic Bax was shown to be decreased, whereas anti-apoptotic Bcl-2 was increased in FLS from RA patients compared with those from osteoarthritis patients (36). Furthermore, an increase in the Bax:Bcl-2 ratio has been demonstrated to promote apoptosis (28). As demonstrated in the present study, the expression of Bcl-2 decreased, whereas that of Bax increased, resulting in an elevated Bax:Bcl-2 ratio, suggesting that matrine promoted the apoptosis of FLS from rats with CIA.

It is well-known that apoptosis requires the activation of a series of caspases, including initiator and effector caspases (33). Following caspase activation, an increasing number of cellular substrates, including the DNA repair protein PARP, are degraded or cleaved, resulting in cell death (28). The activation of caspase-3 plays a crucial role in the initiation of apoptosis (33). A significant decrease in procaspase-3 and -9 expression and a significant increase in caspase-3 and -9 expression were observed in RA-induced FLS apoptosis (37). Accordingly, we also found that matrine significantly induced the activation of caspase-3 in FLS from rats with CIA.

The JAK/STAT signal transduction pathway is activated by many cytokines and growth factors that regulate gene expre- ssion, cell proliferation and differentiation (11). Suppression of JAK/STAT activity leads to the induction of an apoptotic response (38). FLS in the intimal lining in RA have been demonstrated to express high levels of STAT1 (10), and STAT3 expression was found to be elevated in the synovial lining in RA and in an experimental arthritis model (39). Persistent activation of STAT3 contributes to the expression of anti-apoptotic molecules that restrain the induction of programmed cell death (40), whereas STAT3 blockade promotes apoptosis in RA-FLS (9). Previous studies have provided evidence that the JAK2/STAT1/3 signaling pathway may be the upstream mechanism by which FLS proliferation is controlled (12). Studies have reported that matrine suppresses proliferation and induces apoptosis in human cholangiocarcinoma cells by blocking JAK2/STAT3 signaling (17). The appearance of RA-FLS with tumor-like aggressive phenotypes (41) may also justify the use of tumor therapies for RA. We found that phosphorylation of JAK, STAT1 and STAT3 were suppressed by matrine in FLS from rats with CIA, suggesting that matrine induced apoptosis via suppression of JAK2/STAT1/3 signaling.

In conclusion, the results of this study demonstrate that matrine has potent anti-proliferative and pro-apoptotic effects on FLS. Notably, matrine treatment was clinically beneficial in a rat model of arthritis that recapitulates human pathology. Therefore, matrine may represent a novel therapeutic agent for RA that counters the activation of JAK2/STAT1/3 signaling.

\section{Acknowledgements}

This study was supported by the National Natural Science Foundation of China (no. 80360574). The authors would like to thank Professor Qiumei Dong and Sangon Biotech Co., Ltd. for the technical assistance.

\section{References}

1. Zhang W, Zhu J, Du Z, Yu J, Xu Y and Wang F: Intraarticular gene transfer of SPRY2 suppresses adjuvant-induced arthritis in rats. Appl Microbiol Biotechnol 99: 6727-6735, 2015.

2. Liu H, Yang Y, Cai X, Gao Y, Du J and Chen S: The effects of arctigenin on human rheumatoid arthritis fibroblast-like synoviocytes. Pharm Biol 53: 1118-1123, 2015.

3. Tamai M, Kawakami A, Tanaka F, Miyashita T, Nakamura H, Iwanaga N, Izumi Y, Arima K, Aratake K, Huang M, et al: Significant inhibition of TRAIL-mediated fibroblast-like synovial cell apoptosis by IFN-gamma through JAK/STAT pathway by translational regulation. J Lab Clin Med 147: 182-190, 2006.

4. Zong M, Lu T, Fan S, Zhang H, Gong R, Sun L, Fu Z and Fan L: Glucose-6-phosphate isomerase promotes the proliferation and inhibits the apoptosis in fibroblast-like synoviocytes in rheumatoid arthritis. Arthritis Res Ther 17: 100, 2015.

5. Huang M, Zeng S, Qiu Q, Xiao Y, Shi M, Zou Y, Yang X, Xu H and Liang L: Niclosamide induces apoptosis in human rheumatoid arthritis fibroblast-like synoviocytes. Int Immunopharmacol 31: 45-49, 2016.

6. Coskun M, Salem M, Pedersen J and Nielsen OH: Involvement of JAK/STAT signaling in the pathogenesis of inflammatory bowel disease. Pharmacol Res 76: 1-8, 2013.

7. Seavey MM and Dobrzanski P: The many faces of Janus kinase. Biochem Pharmacol 83: 1136-1145, 2012.

8. Ghoreschi K, Laurence A and O'Shea JJ: Selectivity and therapeutic inhibition of kinases: to be or not to be? Nat Immunol 10: 356-360, 2009.

9. Kim SK, Park KY, Yoon WC, Park SH, Park KK, Yoo DH and Choe JY: Melittin enhances apoptosis through suppression of IL-6/sIL-6R complex-induced NF-kB and STAT3 activation and Bcl-2 expression for human fibroblast-like synoviocytes in rheumatoid arthritis. Joint Bone Spine 78: 471-477, 2011. 
10. Kasperkovitz PV, Verbeet NL, Smeets TJ, van Rietschoten JG, Kraan MC, van der Pouw Kraan TC, Tak PP and Verweij CL: Activation of the STAT1 pathway in rheumatoid arthritis. Ann Rheum Dis 63: 233-239, 2004.

11. Krause A, Scaletta N, Ji JD and Ivashkiv LB: Rheumatoid arthritis synoviocyte survival is dependent on Stat3. J Immunol 169: 6610-6616, 2002.

12. Wang H, Fang Y, Wang Y, Wang Z, Zou Q, Shi Y, Chen J and Peng D: Inhibitory effect of curcumol on Jak2-STAT signal pathway molecules of fibroblast-like synoviocytes in patients with rheumatoid arthritis. Evid Based Complement Alternat Med 2012: 746426, 2012

13. Culshaw S, McInnes IB and Liew FY: What can the periodontal community learn from the pathophysiology of rheumatoid arthritis? J Clin Periodontol 38 (Suppl 11): 106-113, 2011.

14. Lee WS, Lim JH, Sung MS, Lee EG, Oh YJ and Yoo WH: Ethyl acetate fraction from Angelica sinensis inhibits IL-1 $\beta$-induced rheumatoid synovial fibroblast proliferation and COX-2, PGE2, and MMPs production. Biol Res 47: 41, 2014.

15. Xin W, Huang C, Zhang X, Xin S, Zhou Y, Ma X, Zhang D, Li Y, Zhou S, Zhang D, et al: Methyl salicylate lactoside inhibits inflammatory response of fibroblast-like synoviocytes and joint destruction in collagen-induced arthritis in mice. $\mathrm{Br} \mathrm{J}$ Pharmacol 171: 3526-3538, 2014.

16. Wu Y,Wang Y,Zhang Y,Chen LP and Wang JY: Effect of matrine on NO and ADMA metabolism pathways in serum and tissues of mice with lipopolysaccharide-induced intestine tissue inflammation. Zhongguo Zhong Yao Za Zhi 39: 2318-2321, 2014 (In Chinese).

17. Yang N, Han F, Cui H, Huang J, Wang T, Zhou Y and Zhou J: Matrine suppresses proliferation and induces apoptosis in human cholangiocarcinoma cells through suppression of JAK2/STAT3 signaling. Pharmacol Rep 67: 388-393, 2015.

18. Sun N, Wang ZW, Wu CH, Li E, He JP, Wang SY, Hu YL, Lei HM and Li HQ: Antiviral activity and underlying molecular mechanisms of matrine against porcine reproductive and respiratory syndrome virus in vitro. Res Vet Sci 96: 323-327, 2014.

19. Yu JL, Li JH, Chengz RG, Ma YM, Wang XJ and Liu JC: Effect of matrine on transforming growth factor $\beta 1$ and hepatocyte growth factor in rat liver fibrosis model. Asian Pac J Trop Med 7: 390-393, 2014

20. Zhou Y, Wu Y, Deng L, Chen L, Zhao D, Lv L, Chen X, Man J, Wang Y, Shan H, et al: The alkaloid matrine of the root of Sophora flavescens prevents arrhythmogenic effect of ouabain. Phytomedicine 21: 931-935, 2014.

21. Fu Q, Wang J, Ma Z and Ma S: Anti-asthmatic effects of matrine in a mouse model of allergic asthma. Fitoterapia 94: 183-189, 2014.

22. Liu N, Kan QC, Zhang XJ, Xv YM, Zhang S, Zhang GX and Zhu L: Upregulation of immunomodulatory molecules by matrine treatment in experimental autoimmune encephalomyelitis. Exp Mol Pathol 97: 470-476, 2014.

23. Yuan X, Garrett-Sinha LA, Sarkar D and Yang S: Deletion of IFT20 in early stage T lymphocyte differentiation inhibits the development of collagen-induced arthritis. Bone Res 2: 14038, 2014

24. Caplazi P, Baca M, Barck K, Carano RA, DeVoss J, Lee WP, Bolon B and Diehl L: Mouse models of rheumatoid arthritis. Vet Pathol 52: 819-826, 2015.

25. Ota F, Maeshima A, Yamashita S, Ikeuchi H, Kaneko Y, Kuroiwa T, Hiromura K, Ueki K, Kojima I and Nojima Y: Activin A induces cell proliferation of fibroblast-like synoviocytes in rheumatoid arthritis. Arthritis Rheum 48: 2442-2449, 2003.

26. Zhang WD, Wang PM, Han RX and Zhang TS: Efficacy and safety evaluation of fire needling for rats with rheumatoid arthritis. Zhongguo Zhen Jiu 33: 334-338, 2013 (In Chinese).
27. Baharav E, Mor F, Halpern M and Weinberger A: Lactobacillus GG bacteria ameliorate arthritis in Lewis rats. J Nutr 134: 1964-1969, 2004.

28. Yan C, Kong D, Ge D, Zhang Y, Zhang X, Su C and Cao X: Mitomycin $\mathrm{C}$ induces apoptosis in rheumatoid arthritis fibroblast-like synoviocytes via a mitochondrial-mediated pathway. Cell Physiol Biochem 35: 1125-1136, 2015.

29. Zheng CJ, Zhao XX, Ai HW, Lin B, Han T, Jiang YP, Xing X and Qin LP: Therapeutic effects of standardized Vitex negundo seeds extract on complete Freund's adjuvant induced arthritis in rats. Phytomedicine 21: 838-846, 2014

30. Wang M, Li K, Nie Y, Wei Y and Li X: Antirheumatoid arthritis activities and chemical compositions of phenolic compounds-rich fraction from Urtica atrichocaulis, an endemic plant to China. Evid Based Complement Alternat Med 2012: 818230, 2012.

31. Shao Q, Zhao X and Yao L: Matrine inhibits the growth of retinoblastoma cells (SO-Rb50) by decreasing proliferation and inducing apoptosis in a mitochondrial pathway. Mol Biol Rep 41: 3475-3480, 2014

32. Shi M, Cui F, Liu AJ, Ma HJ, Cheng M, Song SX, Yuan F, Li DP and Zhang Y: The protective effects of chronic intermittent hypobaric hypoxia pretreatment against collagen-induced arthritis in rats. J Inflamm (Lond) 12: 23, 2015.

33. Luo Y, Wei Z, Chou G, Wang Z, Xia Y and Dai Y: Norisoboldine induces apoptosis of fibroblast-like synoviocytes from adjuvant-induced arthritis rats. Int Immunopharmacol 20: 110-116, 2014.

34. Correia C, Lee SH, Meng XW, Vincelette ND, Knorr KL, Ding H, Nowakowski GS, Dai H and Kaufmann SH: Emerging understanding of Bcl-2 biology: implications for neoplastic progression and treatment. Biochim Biophys Acta 1853: 1658-1671, 2015.

35. Bartok B and Firestein GS: Fibroblast-like synoviocytes: key effector cells in rheumatoid arthritis. Immunol Rev 233: 233-255, 2010.

36. Lee SY, Kwok SK, Son HJ, Ryu JG, Kim EK, Oh HJ, Cho ML, Ju JH, Park SH and Kim HY: IL-17-mediated Bcl-2 expression regulates survival of fibroblast-like synoviocytes in rheumatoid arthritis through STAT3 activation. Arthritis Res Ther 15 R31, 2013.

37. Jie L, Du H, Huang Q, Wei S, Huang R and Sun W: Tanshinone IIA induces apoptosis in fibroblast-like synoviocytes in rheumatoid arthritis via blockade of the cell cycle in the G2/M phase and a mitochondrial pathway. Biol Pharm Bull 37: 1366-1372, 2014.

38. Yeh CT, Huang WC, Rao YK, Ye M, Lee WH, Wang LS, Tzeng DT, Wu CH, Shieh YS, Huang CY, et al: A sesquiterpene lactone antrocin from Antrodia camphorata negatively modulates JAK2/STAT3 signaling via microRNA let-7c and induces apoptosis in lung cancer cells. Carcinogenesis 34: 2918-2928, 2013.

39. Shouda T, Yoshida T, Hanada T, Wakioka T, Oishi M, Miyoshi K, Komiya S, Kosai K, Hanakawa Y, Hashimoto K, et al: Induction of the cytokine signal regulator SOCS3/CIS3 as a therapeutic strategy for treating inflammatory arthritis. J Clin Invest 108: 1781-1788, 2001

40. Liu H and Pope RM: The role of apoptosis in rheumatoid arthritis Curr Opin Pharmacol 3: 317-322, 2003

41. Li GF, Qin YH and Du PQ: Andrographolide inhibits the migration, invasion and matrix metalloproteinase expression of rheumatoid arthritis fibroblast-like synoviocytes via inhibition of HIF-1a signaling. Life Sci 136: 67-72, 2015. 